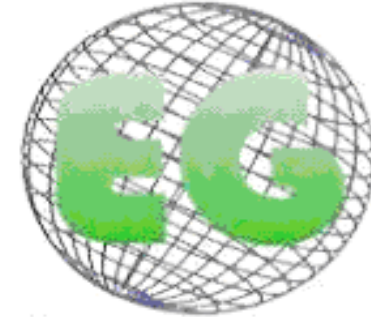

ISSN 1695-6141

$\mathrm{N}^{\circ} 21$

\title{
MODELO DE HOSPITALIZACIÓN Y FRECUENCIA DE REINGRESO EN PACIENTES CON EXACERBACIÓN DE EPOC
}

HOSPITALIZATION MODEL AND THE READMISSION FREQUENCY IN PATIENTS WITH COPD EXACERBATION

*Maciá Soler, L., "*Latour Pérez, J., ${ }^{* * *}$ Moncho Vasallo, J., ${ }^{* * * *}$ Mariscal Crespo, MI., ${ }^{* * * * *}$ Orts Cortés, Ml.

*PhD. Profesora TU Responsable Estudios de Enfermería. Universitat Jaume I de Castellón **PhD. Jefe de Servicio Unidad de Cuidados Intensivos Hospital General de Elche. ${ }^{* * *} \mathrm{PhD}$. Profesor TU de Bioestadística. Universidad de Alicante. ${ }^{* * * * P h D}$. Profesora TU. Directora Máster en Ciencias de la Enfermería. Universidad de Huelva. ${ }^{* * * * *}$ Master Degree. Profesora de Metodología de Investigación. Universidad de Alicante.

\section{Palabras clave: EPOC. Modelo de hospitalización. Reingreso. Mortalidad. Efectividad.}

Keywords: Pulmonary Disease, Chronic Obstructive. Model of hospitalization. Patient readmission, Mortality. Effectiveness

\section{RESUMEN}

Objetivo. Evaluar la efectividad de (UCE) en pacientes con (EPOC) agudizada frente a (HC) en términos de reingreso y mortalidad.

Método. Estudio de cohortes retrospectivo no aleatorizado de pacientes hospitalizados por exacerbación de EPOC durante 2004 en un hospital general. El seguimiento se prolongó hasta el 31 de diciembre de 2006. La censura de datos se produce en la fecha final de seguimiento o aparición de muerte o reingreso. El análisis estadístico se basó en Kaplan-Meier, test del log-rank y regresión de Cox.

Resultados. De los 543 pacientes incluidos, 252 ingresaron en UCE y 291 en HC. Los pacientes de UCE eran mayores $(75,4$ frente a 71,7 años; $p<0,001)$, y tendencia a un menor I. Charlson $(0,46$ frente a 0,$58 ; p<0,07$ ) que los pacientes de HC. La estancia media fue de 3,2 días en UCE frente a 8,9 días en $\mathrm{HC}(\mathrm{p}<0,001)$. En el análisis univariante, los pacientes ingresados en UCE mostraron una mayor incidencia de muerte o reingreso (Riesgo Relativo $[R R] 1,31 ; p<0,001$ ), a expensas de los reingresos ( $R R 1,53 ; p=0,013$ ), no hubo diferencias de mortalidad ( $R R 0,82 ; p=0,34)$. Al ajustar para las covariables del modelo de hospitalización mediante regresión de Cox, estas estimaciones no cambiaron El análisis de las curvas de supervivencia demostró que las diferencias no se debieron a un aumento de los reingresos precoces.

Conclusiones. En el estudio, los pacientes con exacerbación de EPOC ingresados en UCE tuvieron peores resultados en términos de reingreso que los de $\mathrm{HC}$ 


\section{ABSTRACT}

Objective: To evaluate the effectiveness of the Short Stay Unit (USS) in patients who suffer from a chronic obstructive pulmonary disease (COPD) that worsens versus conventional hospitalization $(\mathrm{CH})$ in terms of readmission and mortality.

Patient and Method. Non randomized retrospective study of cohorts of patients hospitalized due to exacerbation of EPOC during 2004 in a general hospital. The monitoring was prolonged until December 31st 2006 or the occurrence of an event (death or readmission). The statistical analysis was based on the Kaplan-Meier method, the log-rank test and Cox regression.

Results. Of the 543 subjects, 252 were admitted in USS and 291 in $\mathrm{CH}$. Those admitted in USS were more advanced in years (75.4 versus 71.7 years; $p<0,001)$, and with a tendency to a smaller Charlson index $(0.46$ versus $0.58 ; p<0,07)$ than the patients in $\mathrm{HC}$. The average stay was 3.2 days in UCE versus 8.9 days in $\mathrm{CH}(\mathrm{p}<0,001)$. In the univariant analysis, the patients admitted in USS showed a major incidence of death or readmission (Relative Risk [RR] 1.31; $p<0,001$ ), at the expense of the readmission (RR 1.53; $p=0,013$ ), while there were no differences in mortality (RR $0,82 ; p=0,34)$. After applying the Cox regression for the hospitalization covariables fit, there was no substantial change in the estimates. The analysis of the survival curves demonstrated that the differences were not due to an increase in precocious readmissions.

Conclusions. In the field of the study, the patients with exacerbation of COPD admitted in USS had worse results in readmission terms than the patients admitted in $\mathrm{CH}$.

\section{INTRODUCCIÓN}

La limitación de camas para hospitalización, aparición de nuevos tratamientos y enfermedades junto con la necesidad de contener el gasto sanitario, ha generado buscar alternativas al modelo de hospitalización convencional (HC). Desde los responsables de gestión se tiende a utilizar medidas que ajusten, el tiempo de hospitalización necesario para cada proceso evitando estancias innecesarias. En esta línea se han desarrollado los hospitales de día (HD), las unidades de hospitalización domiciliaria (UHD) o las unidades de corta estancia (UCE). Al identificarse la estancia como uno de los determinantes principales del gasto sanitario, parece que su reducción sería un factor que incrementaría la eficiencia de los procesos hospitalarios, siempre que se garantice la calidad ${ }^{1}$ de los mismos.

Sin embargo, las decisiones sobre modelos de hospitalización a utilizar en cada caso parece pertinente completarlas con indicadores que relacionen el impacto que supone a medio 0 largo plazo en la vida de los pacientes. Indicadores como el reingreso o la calidad de vida, aportarían más información sobre los modelos de hospitalización a utilizar en cada grupo de pacientes.

Las (UCE) reducen la estancia de hospitalización y si bien, está demostrada su eficiencia en estos términos $^{2}$, están en discusión los criterios de idoneidad de pacientes asignados, al no existir consenso sobre el perfil de pacientes ajustado a este modelo de hospitalización, variando el tipo de usuario de las UCE en cada hospital.

Los pacientes afectados por Enfermedad Pulmonar Obstructiva Crónica (EPOC) constituyen uno de los grupos con mayor reingreso hospitalario de origen multicausal ${ }^{3}$ a lo largo de su vida, llegando a ser en algunos hospitales españoles el $50 \%$ del total de ingresos en UCE. ${ }^{4}$ Entre las causas asociadas al reingreso se encuentran, el estadío de la enfermedad, las complicaciones asociadas ${ }^{5}$, condiciones socioeconómicas 0 estado nutricional ${ }^{6}$. Sin embargo, los estudios encontrados que relacionen como variable predictora de reingreso el modelo de hospitalización se aproximan a un modelo de unidades extrahospitalarias específicas de neumología para este tipo de pacientes ${ }^{7,8,9}$ 
El objetivo del estudio es comparar las diferencias entre dos modelos de hospitalización Corta estancia (UCE) frente al modelo de hospitalización convencional (HC) en pacientes con (EPOC) agudizada, medida en términos de reingreso y mortalidad.

\section{MÉTODO}

\section{Diseño}

Estudio de cohortes restrospectivo basado en datos de registro hospitalario (CMBD).

\section{Pacientes}

Se incluyeron en el estudio todos los pacientes residentes en el área de influencia del Hospital de Elche con 300.000 habitantes que padecieron al menos un episodio de exacerbación de EPOC que requirió hospitalización en 2004. El diagnostico inicial exacerbación de EPOC se realiza en el servicio de urgencias del hospital por el médico de urgencias al ingreso con criterios clínicos y el diagnostico al alta, a partir del informe confirmándose o no el diagnóstico de ingreso. Los informes de alta permiten en la unidad de admisión agrupar a los pacientes por diagnóstico principal y secundarios en Grupos relacionado con el diagnostico (GRD). En el caso del estudio, se incluyen los pacientes que al alta se agrupan en GRD 088 EPOC. La decisión de ingresar en UCE o Neumología (HC) queda a juicio clínico, sin ajustarse a protocolo, ya que el ingreso no se puede aleatorizar por distintos factores entre los que se destaca la disponibilidad de camas. El alta se produce con la mejora del estado del paciente sin valores espirométricos.

Se excluyeron del estudio los pacientes que ingresaron en la unidad de cuidados intensivos (UCI). Los pacientes fueron seguidos durante los tres años del estudio a través del sistema de información del hospital (programa HIGIA) y del Registro de Mortalidad de la Comunidad Valenciana.

La unidad de Neumología tiene 30 camas y está llevada por especialistas en Neumología que comparten actividad con otras unidades hospitalarias y la Unidad de corta estancia la coordinan 4 especialistas en Medicina Familiar y Comunitaria. UCE es un servicio de 30 camas que atiende pacientes con distintas patologías que al ingreso no parecen requerir una hospitalización superior a 48 horas. Si se prolonga la hospitalización pasan a otra unidad.

\section{Variables a estudio}

Entre las variables dependientes se incluyeron, la edad, sexo, zona básica de salud, comorbilidad (índice de Charlson), días de estancia, el posible reingreso antes del tercer año de seguimiento (31 de Diciembre de 2006) y la defunción en caso de existir. La variable independiente contemplada en el estudio la constituye el modelo de hospitalización. La HC agrupó cualquier estancia en los pacientes objeto de estudio en las unidades de Medicina Interna y Neumología. Las variables respuesta estudiadas fueron el tiempo hasta el fallecimiento, reingreso o el desenlace combinado de reingreso y muerte en la estancia o después del alta y antes del 31 de Diciembre de 2006. 


\section{Análisis de datos}

Tras un análisis descriptivo, se realizó un análisis univariante mediante pruebas paramétricas $t$ (prueba $t$ para muestras independientes 0 ANOVA para variables cuantitativas y test ji-cuadrado o test de Fisher para datos categóricos). El análisis de supervivencia univariante se realizó mediante Kaplan-Meier y la comparación de curvas con el test del log-rank. Para intentar controlar el posible efecto de confusión se realizó un análisis multivariante mediante un modelo de regresión de Cox con covariables fijas. La posible interacción entre el modelo de hospitalización y las covariables se analizó mediante la incorporación en el modelo de Cox de un término de interacción con cada una de ellas. La asunción básica del modelo (riesgos proporcionales) se comprobó mediante un análisis gráfico de las funciones de riesgo.

Todos los contrastes de hipótesis fueron bilaterales, y con un nivel de significación de $\mathrm{p}<0,05$.

El análisis se realizó con el programa Stats Direct.

\section{RESULTADOS}

De los 543 pacientes incluidos, 251 ingresaron en UCE y 293 en HC. El grupo de pacientes ingresados en UCE presentó una mayor edad media, (75,4 años frente a 71,7 años; $p<$ $0,001)$ pero un menor índice de Charlson $(0,46$ frente a 0,$58 ; p<0,07)$ que los pacientes de HC. La distribución de los pacientes en función de la zona básica de salud a la que pertenecen y el tipo de hospitalización fue relativamente similar y no ofreció diferencias significativas. La estancia media fue de 3,2 días en la UCE frente a 8,9 días en planta ( $p$ $<0,001)$

Tabla 1. Características de los participantes según modelo de hospitalización

\begin{tabular}{llll}
\hline Variable & $\begin{array}{l}\text { HC } \\
(\mathrm{n}=293)\end{array}$ & $\begin{array}{l}\text { UCE } \\
(\mathrm{n}=250)\end{array}$ & Valor $\mathbf{p}$ \\
\hline Edad (años, media $\pm \mathrm{DE}[\mathrm{IC95}])$ & $72 \pm 9,4[70,5-72,9]$ & $75,4 \pm 10,7[74,3-76,6]$ & $<0,001$ \\
Sexo (mujeres/hombres) & $30 / 263$ & $34 / 216$ & 0,226 \\
Índice de Charlson (media $\pm \mathrm{DE}[\mathrm{IC} 95])$ & $0,58 \pm 0,9[0,48-0,68]$ & $0,46 \pm 0.8[0,38-0,54]$ & 0,07 \\
Estancia (días, media $\pm D E[$ IC95]) & $8,9 \pm 0,4[8,0-9,7]$ & $3,2 \pm 0,1[3,1-3,4]$ & $<0,001$ \\
\hline \multicolumn{2}{c}{ HC: Hospitalización convencional; UCE: Unidad de corta estancia; IC: Intervalo de confianza; DE: desviación estándar }
\end{tabular}

HC: Hospitalización convencional; UCE: Unidad de corta estancia; IC: Intervalo de confianza; DE: desviación estándar

Las comorbilidades más frecuentes (más de 10 casos) fueron tuberculosis respiratoria o sin especificar; hiperglucemia; hipertensión arterial; historia de infarto agudo de miocardio; fibrilación auricular; insuficiencia cardiaca congestiva; bronquiectasia con exacerbación aguda; fracaso respiratorio y fracaso respiratorio reagudizado.

En el análisis univariante, los pacientes ingresados en UCE mostraron una mayor incidencia del evento combinado muerte o reingreso (Riesgo Relativo [RR] 1,31; $p<0,013$ ), a expensas de los reingresos ( $R R$ 1,53; $p=0,001$ ), mientras que no hubo diferencias de mortalidad ( $R R$ $0,82 ; p=0.34)$. 
Tabla 2. Análisis univariante del reingreso, fallecimiento y reingreso-fallecimiento posterior al alta.

\begin{tabular}{lrrrrrr}
\hline Variable & $\begin{array}{r}\text { HC } \\
\mathbf{n = 2 9 3}\end{array}$ & $\begin{array}{r}\text { UCE } \\
\mathbf{n = 2 5 0}\end{array}$ & Valor $\mathbf{p}$ & $\begin{array}{c}\text { Hazard } \\
\text { Ratio }\end{array}$ & IC95\% & $\mathbf{p}$ \\
\hline Reingreso & $129(44,0 \%)$ & $151(60,4 \%)$ & $<0,001$ & 1,53 & $1,2-1,9$ & $<0,001$ \\
Fallecimiento & $54(18,4 \%)$ & $40(16,0 \%)$ & 0,456 & 0,82 & $0,5-1,2$ & 0,34 \\
Reingreso o fallecimiento & $163(55,6 \%)$ & $168(67,2 \%)$ & 0,006 & 1,31 & $1,06-1,5$ & 0,013 \\
\hline
\end{tabular}

HC: Hospitalización convencional; UCE: Unidad de corta estancia; IC: Intervalo de confianza

El análisis de las curvas de supervivencia (tiempo hasta el reingreso, muerte o el evento combinado) mostró un aumento progresivo de las diferencias absolutas de riesgo con el tiempo (Figuras 1a,1b y 1c).

Figura 1a. Curvas de supervivencia: muerte o reingreso

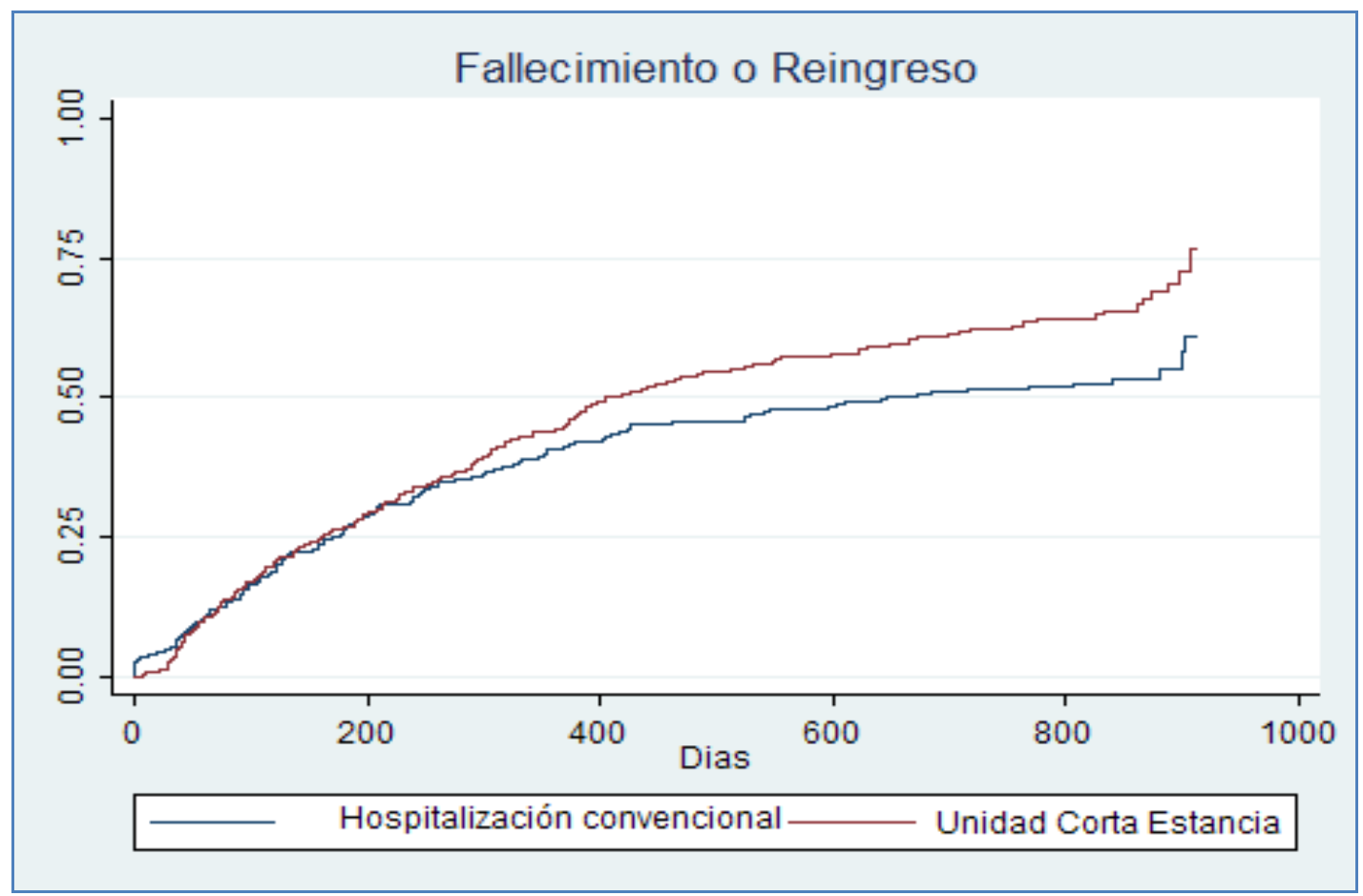


Figura 1b. Curvas de supervivencia: Reingreso

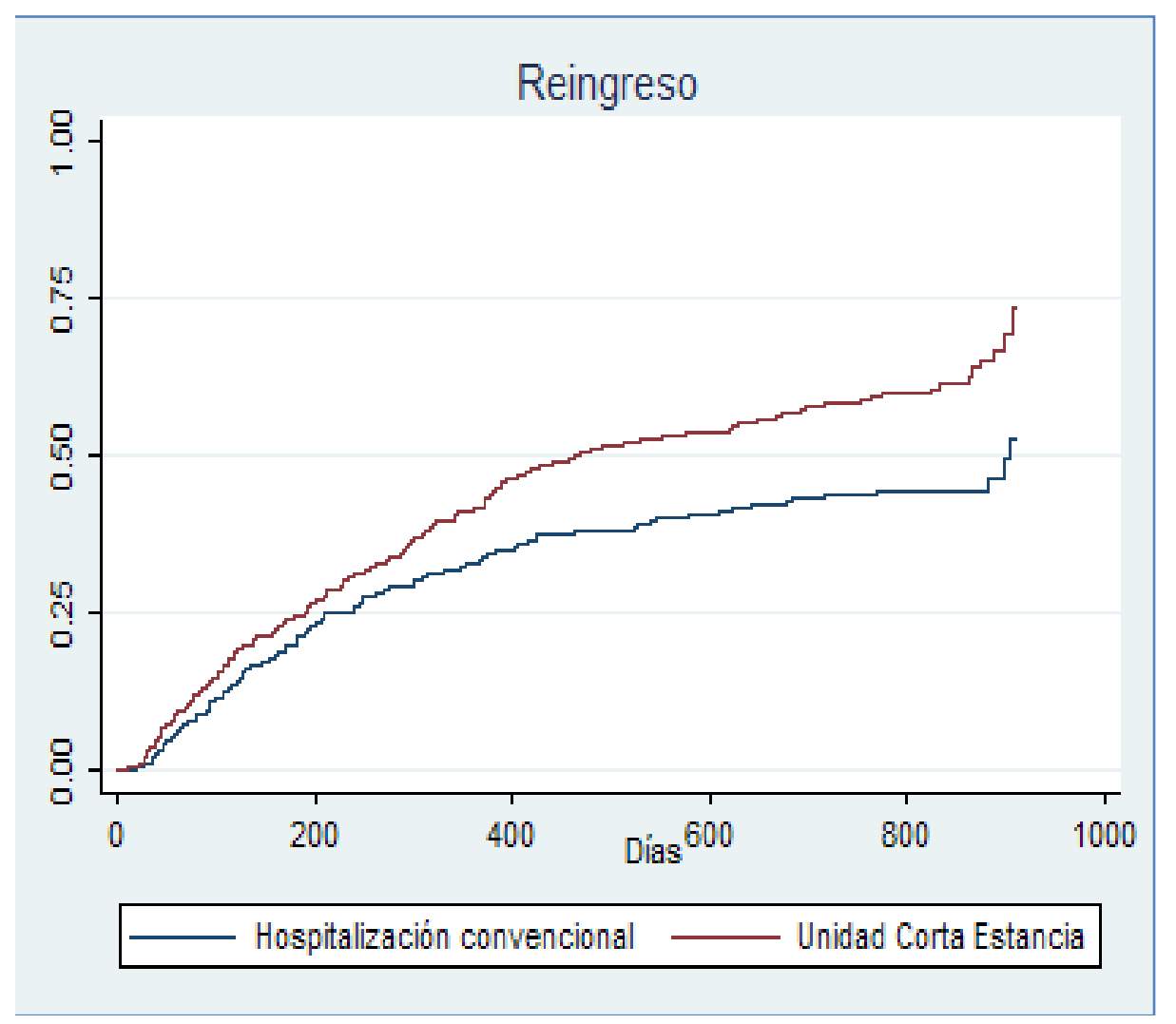

Figura 1c. Curvas de supervivencia: Fallecimiento

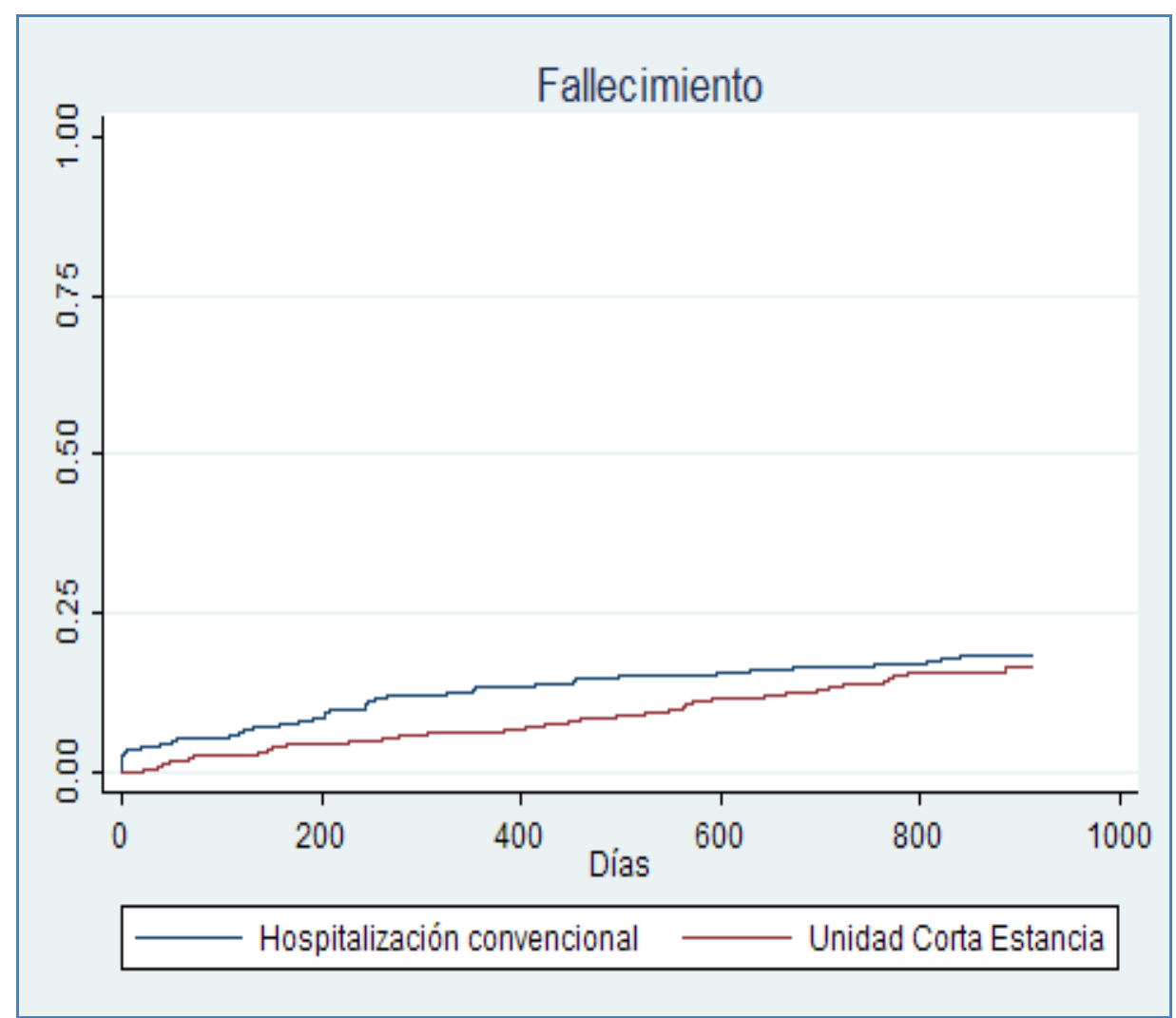


Al ajustar para las covariables del modelo de hospitalización mediante regresión de Cox, estas estimaciones no cambiaron sustancialmente respecto al riesgo estimado a partir del análisis univariante, quedando aislada la variable tipo de hospitalización como única variable predictiva independiente de riesgo entre el grupo de variables consideradas.

Tabla 3. Análisis multivariante, evento combinado (muerte o reingreso)

\begin{tabular}{llc}
\hline Variable Respuesta & $\begin{array}{l}\text { Variables explicativas además del tipo de } \\
\text { hospitalización }\end{array}$ & $\begin{array}{c}\text { Hazard Rate (IC 95\%) } \\
\text { UCE vs HC }\end{array}$ \\
\hline & Ninguna (Análisis crudo) & $1,32(1,06-1,64)$ \\
& Edad (continua) & $1,29(1,03-1,61)$ \\
Muerte-Reingreso & Edad (3 variables dummy) & $1,31(1,05-1,63)$ \\
& Sexo & $1,32(1,06-1,65)$ \\
& Charlson (3 categorías) & $1,33(1,07-1,65)$ \\
& Propensión (4 categorías) & $1,31(1,05-1,63)$ \\
& Edad continua + Sexo + Charlson + Propensión & $1,31(1,05-1,65)$ \\
\hline
\end{tabular}

HC: Hospitalización convencional; UCE: Unidad de corta estancia; IC: Intervalo de confianza

No se encontró interacción entre el modelo de hospitalización y la edad y el índice de Charlson El término de interacción hospitalización-sexo alcanzó una significación límite $(p=0,06)$. En la figura 2 se presentan los resultados de supervivencia crudos estratificados por tipo de hospitalización y sexo.

Figura 2. Interacción modelo de hospitalización, muerte o reingreso y sexo

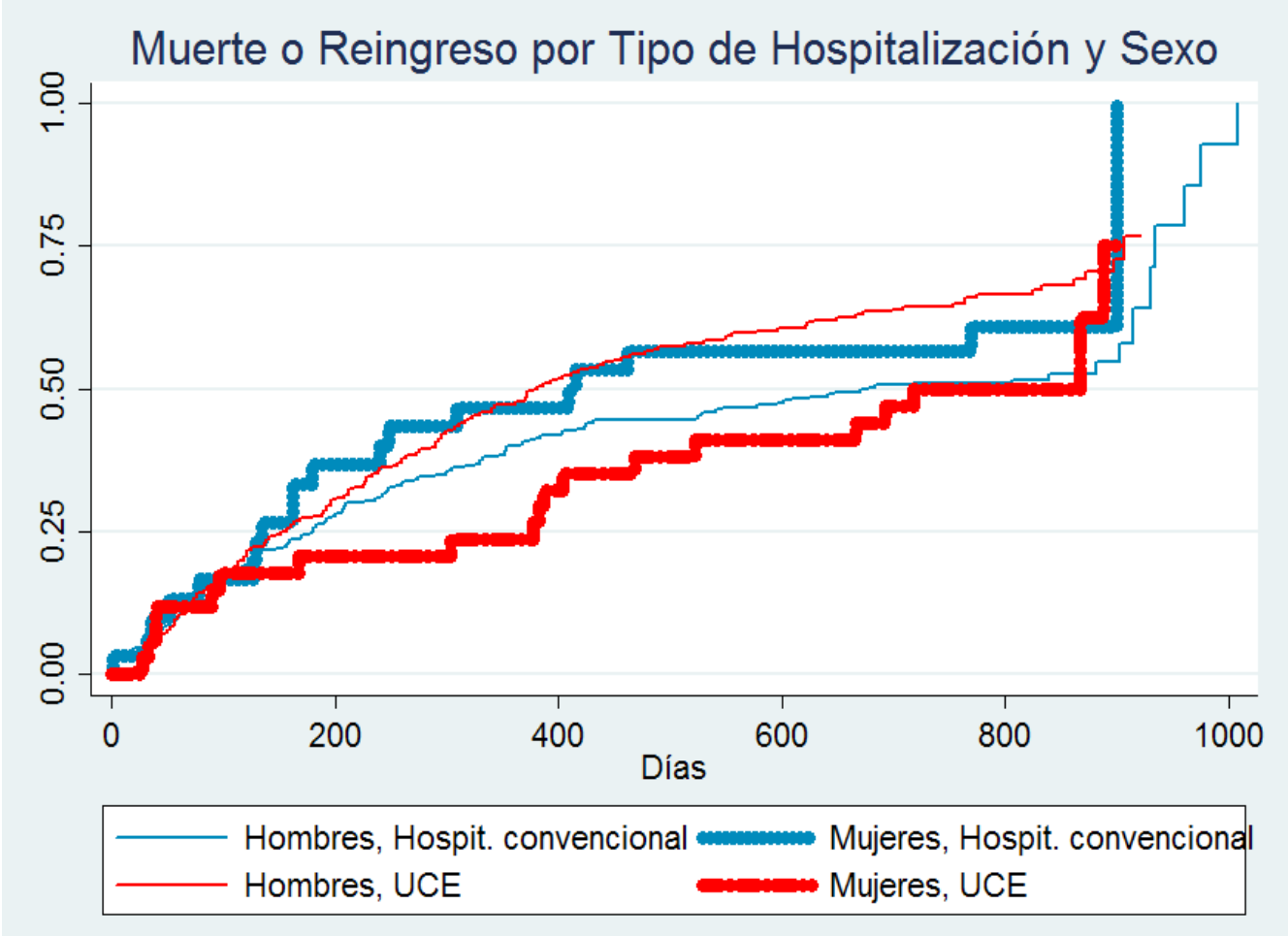


Estos resultados muestran que entre los pacientes ingresados en $\mathrm{HC}$ el riesgo de muerte $\mathrm{o}$ reingreso tiende a ser mayor en las mujeres que en los varones; entre los pacientes ingresados inicialmente en UCE, en cambio, el riesgo de las mujeres tiende a ser menor que el de los varones

\section{DISCUSIÓN}

Los resultados del estudio muestran que las diferencias absolutas en la tasa de reingresos es pequeña al principio y que las diferencias aumentan progresivamente con el tiempo. Este perfil sugiere que los peores resultados de los pacientes ingresados en UCE no son atribuibles a una deficiente calidad asistencial en la UCE. Por otra parte, el análisis multivariante muestra que estas diferencias no parecen atribuibles a la edad, la comorbilidad, el sexo, la estancia o la zona geográfica.

Las mayores limitaciones de este estudio están relacionadas con su naturaleza observacional y retrospectiva. En efecto, dado el limitado número de variables recogido en el CMBD, no se puede descartar que existan variables de confusión importantes que no han sido controladas en el análisis multivariante. EI $\mathrm{FEV}_{1}$ es una variable determinante de estadío del $\mathrm{EPOC}^{8}$ y pronóstico de reingreso, sin embargo, no ha podido utilizarse en el estudio, ya que en UCE no se determina al alta.

Por otro lado, la decisión de desviar el enfermo a la UCE u HC no es aleatoria, ya que la decide el médico de Urgencias. Este efecto se ha intentado examinar en nuestro estudio mediante el uso de análisis de propensión (resultados no presentados). Sin embargo, el valor de este análisis en el estudio es escaso, ya que la capacidad de discriminación del índice de propensión fue muy limitada (baja área bajo la curva ROC).

Un ensayo clínico aleatorizado podría proporcionar una evidencia de mayor calidad sobre la efectividad de la UCE. Sin embargo las dificultades logísticas de su realización y la limitada validez externa de sus resultados lo hacen poco viable.

En caso de confirmarse con estudios posteriores la relación entre el modelo de hospitalización y el riesgo de reingreso, parece pertinente ajustar el perfil de pacientes al modelo de hospitalización para mejorar la efectividad de las unidades. El tiempo entre reingresos se atribuye a estado de los pacientes en el domicilio, educación durante los intervalos entre hospitalización y en definitiva factores de apoyo que podrían evidenciar una necesidad de apoyo socio-sanitario importante, tal como muestran algunos estudios ${ }^{10,11}$, ya que se detectan estados depresivos relacionados con la accesibilidad a tratamientos, dificultades para obtener oxigenoterapia o ausencia de programas de rehabilitación respiratoria, que podrían ser alguna de las causas de reingreso a medio, largo plazo ${ }^{7}$.

Tradicionalmente los pacientes con exacerbación de EPOC cuando requerían hospitalización lo hacían en HC. Las (UCE), comienzan a implantarse como unidades costeefectivas alternativas al modelo $(\mathrm{HC})$ para tratar pacientes con problemas respiratorios como asma, EPOC, y aparecen en el entorno de la Unión Europea durante la década de los 90 del siglo XX. Diferentes sociedades científicas (como SEPAR, SEQ, SEMES, SEMG, SEMERGEN) recomiendan que la estancia de las personas con problemas respiratorios exacerbados, tenga lugar en las UCE .

Si la comparación entre las UCE y la hospitalización convencional se realiza exclusivamente en términos de estancia, resulta evidente que las unidades de corta estancia son más efectivas que la hospitalización convencional. Sin embargo, si se evalúa la efectividad en 
términos de desenlaces relevantes como el reingreso o la mortalidad, las conclusiones pueden ser diferentes.

El estudio aísla la variable modelo de hospitalización como riesgo de reingreso hospitalario en UCE frente a HC., frente a los predictores tratamiento, $\mathrm{FEV}_{1}^{8,12}$ o comorbilidades ${ }^{13}$.

Otras variables predictivas del reingreso, sobre todo después del primer mes y antes de los 6 posteriores al alta, se consideran la calidad de vida en el domicilio, estilos de vida y capacidad para autocuidarse, junto con depresión ocasionada por la dependencia de tabaco o la incapacidad de ser independiente ${ }^{14}$.

Desde UCE, el factor pronóstico de reingreso a los 10 días del alta se asocia, sobre todo, al número de días ingresado el año previo ${ }^{32}$. Sin embargo, en algunos estudios muy incipientes realizados desde unidades de corta estancia, se ha detectado un aumento del reingreso del $9,9 \%$ en UCE frente al $7 \%$ observado en hospitalización convencional ${ }^{15}$, sin asociar posibles causas del mismo.

No existe, en general, un acuerdo en la consideración de reingreso precoz o tardío, si bien parece que se considera precoz hasta 30 días posteriores al ingreso índice; en el estudio los ingresos precoces muestran pequeñas diferencias al alta en los dos modelos de hospitalización y estos aspectos generan dificultades concluyentes que indican, junto con el resto de resultados, la pertinencia de continuidad en la investigación sobre variables predictoras de reingreso de pacientes con EPOC.

\section{AGRADECIMIENTOS:}

El estudio ha sido posible gracias a la facilidad de datos de distintos servicios del Hospital General de Elche; Unidad de Documentación Clínica, dirigida por la Dra. Clara Pérez Cascales, Unidades de Corta Estancia y Neumología y Servicio de Informática.

\section{BIBLIOGRAFÍA}

1. Muiño Miguez A. Unidad médica de corta estancia. Rev. Anales de Med. Interna 2002;19 (5). Editorial.

2. Corbella Xavier, Salazar Albert, Maiques JM, Juan Antoni. Unidad de corta estancia de urgencias como alternativa a la hospitalización convencional. Cartas científicas. Rev. Med.Clin. (Barc) 2002. 118; (13): 515 -6

3. Miravitlles M, Mayordomo C, Artés M, Sánchez-Agudo L, Nicolau F, Segú JF, et al. Treatment of chronic obstructive pulmonary disease and its exacerbations in general practice. Respiratory Medicine J. 1999;93:173-9

4. Juan A, Salazar A, Alvarez A, Perez JR, Garcia L, Corbella X.Effectiveness and safety of an emergency department short-stay unit as an alternative to standard inpatient hospitalisation. Emerg Med J. 2006;23 (11):833-7.

5. C González. E Servera. G Ferris. ML Blasco. J Marín. Factores predictivos de reingreso hospitalario en la agudización de la EPOC moderada-grave Rev. Arch Bronconeumol. 2004;40:502-7.

6. Alcolea Batres S, Villamor León J, Álvarez-Sala R. EPOC y estado nutricional. Rev. Archivos de Bronconeumología. 2007; 43(5):283 - 288.

7. Jimenez Puente A, Fernandez Guerra J, Hidalgo Rojas L, Domingo Gonzalez S, Lara Blanquer A, Garcia Alegria J. Calidad de la asistencia hospitalaria y riesgo de 
reingreso precoz en la exacerbación aguda de la EPOC. Rev.An.Medicina Interna.2003;20(7): $340-346$

8. Vega Reyes J, Montero Pérez-Barquero $M$, Sánchez Guijo $P$, Factores pronósticos de reingreso en la enfermedad_pulmonar_obstructiva_crónica Med Clin (Barc). 2004;122:293-7.

9. Broquetas J, Pedreny R, Martínez-Llorens J, Sellarésa J. Gea J. Unidad neumológica de estancia corta: un nuevo dispositivo asistencial de hospitalización Rev. Arch Bronconeumol. 2008;44:252-6.

10. Garcia-Aymerich J, Hernandez C, Alonso A, Casas A, Rodriguez-Roisin R, Anto JM, Roca J. Effects of an integrated care intervention on risk factors of COPD readmission. Respir Med. 2007; 101:1462-9

11. Polzien G. Practical interventions to prevent or lower emergent care and rehospitalization: patient education for home care patients with COPD. Home Healthc Nurse. 2007; 25:482-5.

12. Ng TP, Niti M, Tan WC, Cao Z, Ong KC, Eng P. Depressive symptoms and chronic obstructive pulmonary disease: effect on mortality, hospital readmission, symptom burden, functional status, and quality of life. Arch Intern Med. 2007; 167:60-7.

13. Martínez Marco E, Aranaz Andrés J. ¿Existe relación entre el reingreso hospitalario y la calidad asistencial? Calidad asistencial. 2002; 17:79-86.

14. Horn SD, Horn RA. Reliability and Validity of the severity of Illness Index. Med Care. 1986;24:159-78.

15. Diz-Lois Palomares MT, de la Iglesia Martínez F, Nicolás Miguel R, Pellicer Vázquez C, Ramos Polledo V, Diz-Lois Martínez F. Predictive factors of unplanned hospital readmission in patients discharged at a short stay medical unit Rev. An Med Interna. 2002;19 (5):221-5. 